\title{
A aplicação dos ostracodes (Crustacea) em pesquisas paleoceanográficas e paleoclimáticas
}

\author{
Cristianini Trescastro Bergue \\ Laboratório de Micropaleontologia \\ Universidade do Vale do Rio dos Sinos (RS) \\ cbergue@unisinos.br
}

RESUMO Os ostracodes constituem um grupo de microfósseis com grande aplicabilidade à geociências, o que é atestado pela contribuição que seu estudo trouxe a paleoecologia e bioestratigrafia. A partir da segunda metade do século XX, os ostracodes revelaram-se também como uma excelente fonte de dados para estudos oceanográficos por meio da relação entre as mudanças nas suas assembléias e mudanças nas características hidrológicas, tais como a temperatura e a salinidade. A presença de uma carapaça quitinocalcítica possibilita ainda a realização de análises geoquímicas, que fornecem dados adicionais sobre eventos oceanográficos e auxiliam a compreender suas influências na biota marinha. Sua pesquisa é atualmente desenvolvida em algumas instituições brasileiras, oferecendo oportunidades para o aprimoramento da paleoceanografia em nosso país.

PALAVRAS-CHAVE paleoceanografia, ostracodes, micropaleontologia, elementostraço, isótopos estáveis

Este documento deve ser referido como segue:

Bergue C.T. 2006. A aplicação dos ostracodes (Crustacea) em pesquisas paleoceanográficas e paleoclimáticas.Terræ Didatica, 2(1):54-66. <http:// www.ige.unicamp.br/ terraedidatica/>
ABSTRACT Ostracodes constitutes a microfossil group with many applications to geosciences as denoted by their paleoenvironmental and biostratigraphic uses. From the middle of 20th century ostracode assemblages began to be used as oceanographic proxies because of the relationship between faunal turnovers and hydrologic changes. The chitino-calcitic shell enables also geochemical analyses which permit the assessment of the influence of climatic events in the oceanic circulation and water properties. That research field has been developed in some Brazilian research institutions, improving the oceanographic knowledge in our country.

KEYWORDS paleoceanography, ostracodes, micropaleontology, trace elements, stable isotopes 


\section{Introdução}

$\mathrm{Na}$ atualidade, uma das principais linhas de investigação na área de geociências refere-se ao estudo das mudanças climáticas globais. A cada ano somam-se evidências de que o clima no planeta sofre variações cíclicas que alteram o ambiente e influenciam diversos setores da sociedade.

$\mathrm{O}$ registro geológico mostra que a Terra passou por mudanças ao longo da sua evolução que constituem aspectos intrínsecos da sua dinâmica. Entretanto, sabe-se que atividades antrópicas, como o desmatamento, a emissão de gases do efeito estufa e de efluentes tóxicos, entre outros, são capazes de influenciar o clima, por atuarem diretamente nos ciclos biogeoquímicos.

Além dos registros pluviométricos e de temperaturas médias anuais, outros indicadores podem ser considerados no estudo das mudanças ambientais. O oceano e a atmosfera formam um sistema integrado que regula o clima terrestre por meio da captação de gás carbônico e distribuição de calor no planeta. Essa interação torna o registro sedimentar das bacias oceânicas uma excelente fonte de dados para o estudo das mudanças ambientais ao longo do tempo (Bradley 1999).

Alguns organismos marinhos são potenciais indicadores dessas mudanças por possuírem esqueletos cuja análise revela algumas propriedades físico-químicas dos oceanos em que viveram. O registro fóssil destes organismos permite ainda o estudo de ambientes oceânicos do passado. Dentre os diferentes grupos de microfósseis explorados com esta finalidade estão os ostracodes (Fig. 1), cujas características gerais e potencialidades para a pesquisa paleoceanográfica serão expostas neste texto.

A difusão do uso de ostracodes neste tipo de pesquisa ocorreu no final da década de 1960, com a ampliação do conhecimento sobre associações de águas profundas e do seu gradiente batimétrico de distribuição (Benson 1969). A esta abordagem faunística somaram-se as análises de elementos-traço e isótopos estáveis, permitindo estudos mais detalhados e com maior resolução (Dwyer et al. 1995, Didiè e Bauch, 2002).

\section{A estrutura dos oceanos modernos}

Os oceanos são sistemas complexos formados por uma série de processos geológicos que modelaram sua fisiografia e as biotas que neles vivem. A circulação, a estrutura hidrológica, o volume de gelo e os processos sedimentares são algumas características que mudaram constantemente ao longo do tempo.

A formação de um oceano se inicia com a lenta ruptura de uma massa continental, cuja separação é acompanhada pela formação do assoalho oceânico a partir de material proveniente do manto. As margens continentais geradas são subdivididas, em termos fisiográficos, em plataforma, talude e sopé, além do qual se situa a planície abissal. Graças aos processos de sedimentação, influenciados pelas características climáticas regionais, é que se formam as bacias sedimentares marginais.

Em termos hidrológicos os oceanos são compostos por massas d'água cujas densidades variam conforme a temperatura e salinidade. Estes corpos são classificados, de acordo com a posição na coluna oceânica, em massas superficiais, intermediárias e de fundo. A porção superior é composta por águas relativamente quentes (temperaturas superiores a 
$10^{\circ} \mathrm{C}$ ), na qual se situa a camada de mistura superficial, cuja espessura varia em diferentes regiões. As camadas subjacentes são mais frias, e compostas por águas que afundam em altas latitudes e migram em direção ao equador (Fig. 2). Bruun (1957) propôs a terminologia utilizada para denominá-las, onde o domínio das águas quentes superiores chama-se termosfera, e o das águas frias, psicrosfera. No limite entre estas duas regiões, aproximadamente entre 300-1000 m, existe uma zona transicional denominada termoclina, a qual está associada a zona de mínimo de oxigênio (ZMO), resultante da depleção de oxigênio por atividade biológica.

Uma das principais conseqüências das mudanças climáticas é a modificação nos processos de circulação oceânica. Isto é explicado pelo fato das massas de fundo como a Água Profunda do Atlântico Norte (APAN) e a Água Antártica de Fundo (AAF), serem formadas pelo afundamento das águas superficiais próximo a regiões polares em decorrência do aumento de densidade. Estas massas circulam por longas distâncias e têm um tempo de residência médio de $1 \mathrm{Ka}$, de forma que modificações em suas propriedades físicas e químicas sofridas durante a formação afetam o transporte de calor global e as características hidrológicas das bacias oceânicas.

\section{Um breve histórico do estudo dos oceanos}

A paleoceanografia estuda as mudanças ocorridas nos oceanos ao longo do tempo geológico, investigando as variações hidrológicas e sedimentares, e seu reflexo nas biotas marinhas. Por questões práticas a humanidade cedo desenvolveu um vínculo muito forte com o oceano. Alguns povos tinham o mar como importante fonte de recursos e desenvolveram uma cultura náutica que influenciou sua formação histórica e gerou os primeiros conhecimentos sobre os organismos marinhos.

Através dos oceanos estabeleceram-se importantes rotas de expansão econômica e de subseqüente difusão de conhecimentos a tal ponto que o domínio de técnicas de navegação representou uma vantagem estratégica. Povos representativos ao longo da história possuíram forte tradição náutica, na qual acumularam conhecimentos sobre diversos fenômenos oceanográficos, como as variações de maré, correntes oceânicas e regimes de ventos.

Até então conhecer o mar constituía uma atividade essencialmente prática, desenvolvida basicamente por navegadores mercantes ou militares. Apenas com o progresso ocorrido nas ciências naturais a partir do final do século XVIII é que teve

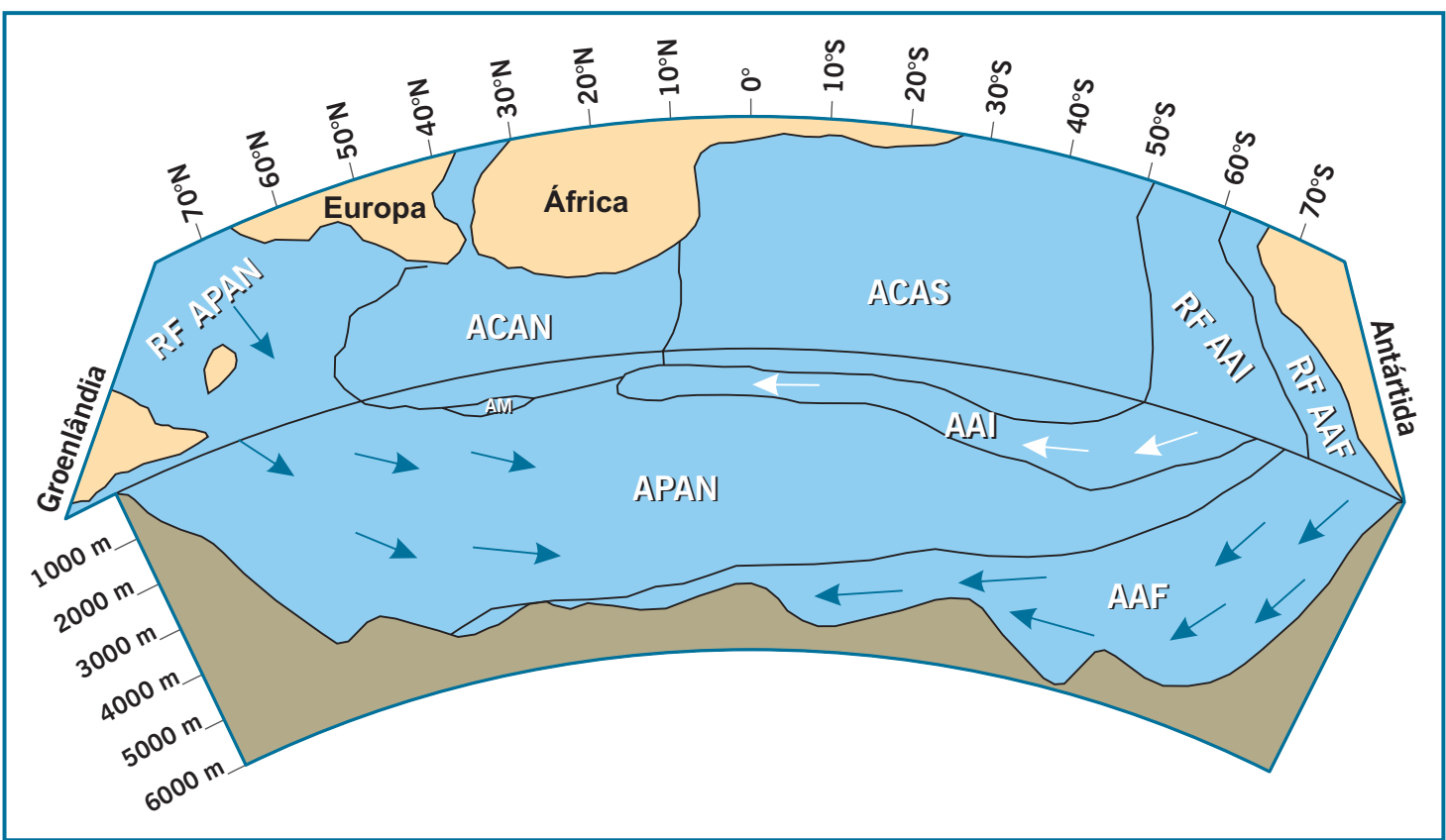

Figura 2 - Representação esquemática do Oceano Atlântico atual mostrando as principais massas d'água e suas regiões de origem. APAN. Água Profunda do Atlântico Norte; AAF. Água Antártica de Fundo; AAI. Água Antártica Intermediária; ACAN. Água central do Atlântico Norte; ACAS. Água Central do Atlântico Sul; AM. Água Mediterrânea; RF APAN. Região de Formação da APAN; RF AAI. Região de Formação da AAI; RF AAF. Região de Formação da AAF (modificado de Dwyer et al. 2002) 
início o estudo do ambiente marinho de forma mais detalhada. Cruzeiros oceanográficos começaram a ser realizados para coleta de dados e medições até que na segunda metade do século XIX a oceanografia tomou um grande impulso com a expedição oceanográfica do navio britânico HMS Challenger, entre 1872 e 1878. O Challenger era um navio com propulsão a vela e vapor adaptado como navio de pesquisa. Nele foram instalados equipamentos de amostragem de superficie e do fundo oceânico, que permitiram a coleta de sedimentos a grandes profundidades, o que constituía algo extraordinário para a época. O material coletado nesta expedição foi estudado por muitos anos e gerou novos conhecimentos em diversas áreas da biologia.

As expedições do navio alemão Meteor (19251927) e do sueco Albatross (1947-1948) constituíram marcos importantes na oceanografia geológica, especialmente pela contribuição que trouxeram para o estudo da sedimentação marinha. O Meteor foi o primeiro navio a realizar perfurações no fundo oceânico, e os dados obtidos pelo Albatross resultaram nas primeiras evidências da ciclicidade da sedimentação marinha. Este último navio foi pioneiro na perfuração de testemunhos a pistão, um sistema de amostragem que permitiu pela primeira vez a obtenção de testemunhos com um mínimo de compactação e perturbação de camadas. Este sistema, com algum aperfeiçoamento, é o mesmo utilizado atualmente em navios oceanográficos (Seibold e Berger 1996).

A oceanografia iniciou uma nova fase no final da década de 1960 com a aplicação dos conceitos da tectônica de placas, melhorando a compreensão sobre a formação da crosta oceânica. Nesta mesma época estabeleceu-se o Deep Sea Drilling Project (DSDP) que constituiu um verdadeiro marco na oceanografia moderna. Este projeto, coordenado por instituições norte-americanas, mas do qual participaram institutos de pesquisa de diferentes países estendeu-se de 1968 até 1983. A partir de 1983 ele passou-se a chamar Ocean Drilling Program (ODP), denominação sob a qual continua suas atividades até os dias de hoje. O primeiro navio utilizado neste projeto, o Glomar Challenger era dotado de um sofisticado sistema de coleta, orientado por meio de satélites, que permitia a perfuração com alta precisão de pontos específicos do fundo oceânico abrangendo intervalos terciários e inclusive cretáceos. Este navio foi substituído pelo JOIDES Resolution que está atualmente em operação realizando o mesmo tipo de trabalho.

\section{Características gerais dos ostracodes}

A classe Ostracoda constitui um grupo de pequenos crustáceos aquáticos que apresenta um registro paleontológico abrangendo praticamente todo o Fanerozóico. O corpo da maioria das espécies é envolto por uma carapaça cujo tamanho varia entre 0,3 e $1 \mathrm{~mm}$ de comprimento. Embora ocorram também em ambientes transicionais e dulciaqüícolas, é nos oceanos que possuem maior diversidade de formas.

São de especial interesse paleontológico pelo amplo registro fóssil (Ordoviciano-Holoceno) e por suas aplicações em estudos paleoambientais, bioestratigráficos e evolutivos. Seu registro fóssil deve-se a composição quitino-calcítica da carapaça composta por duas valvas que se articulam dorsalmente, mudada a medida que o indivíduo se desenvolve. Até atingir a fase adulta os ostracodes passam, em geral, por oito estágios de crescimento (instars), e em cada um deles uma nova carapaça é sintetizada. Diferente de outros crustáceos, o carbonato utilizado para sua síntese não é reabsorvido, provindo totalmente da água.

Tradicionalmente, os ostracodes são pesquisados por paleontólogos o que explica o fato da carapaça, em geral a única parte fossilizável, ser um importante tema de estudos. Esta estrutura, secretada pela epiderme, é estruturada de forma complexa e diversificada, existindo terminologia detalhada para descrevê-la (Fig. 1). É nas características morfológicas que se baseia a sistemática das espécies fósseis e grande parte das atuais.

Estruturas presentes na superfície das carapaças, denominadas ornamentações (espinhos, costelas, tubérculos, reticulações, entre outras), foram estudadas por diferentes autores sob o aspecto funcional. A partir de análises morfométricas, Benson (1972) propôs que as ornamentações estariam organizadas de forma a otimizar a resistência mecânica, equacionando a disponibilidade de matéria-prima com a funcionalidade da carapaça. Estas idéias foram complementadas por Swanson (1995) que sustentou que a ornamentação constituiria um sistema de proteção contra a dissolução. Como é sabido, com o aumento da profundidade as águas oceânicas tornam-se gradativamente mais pobres em carbonato. Este desequilíbrio promove um incremento na dissolução das carapaças, até atingir-se uma isóbata (aproximadamente $3.000 \mathrm{~m}$ ) na qual a precipitação biológica dos carbonatos 
torna-se inviável, denominada zona de compensação da calcita (CCD - Calcite Compensation Depth) (Armstrong e Brasier, 2005).

Em conseqüência disso, organismos com esqueletos carbonáticos que habitam regiões próximas ou abaixo da CCD necessitam adaptações para este estado de subsaturação. No caso dos ostracodes, detritos carbonáticos (oriundos do fitoplâncton, por exemplo) aprisionados na ornamentação afastam a zona de insaturação (Fig. 3). Alguns tipos de ornamentação, portanto, desempenharam uma função adaptativa que auxiliou o estabelecimento de uma fauna psicrosférica de ostracodes. Em um estudo recente, Hunt e Roy (2006) discutem ainda como estes eventos teriam modulado a evolução do grupo, com base em medições de diferentes espécies do gênero Poseidonamicus, um clássico habitante de águas profundas, ao longo do Cenozóico.

\section{Distribuição dos ostracodes marinhos}

O padrão de distribuição geográfica dos ostracodes marinhos é o resultado de processos de vicariância, dispersão, evolução e extinção, decorrentes de eventos tectônicos e/ou climáticos. Uma vez que estes eventos afetam a circulação e formação das massas d'água, influenciam diretamente as espécies.

Além das características hidrológicas, os sedimentos de fundo são determinantes na diversidade e abundância dos ostracodes. Coimbra et al.

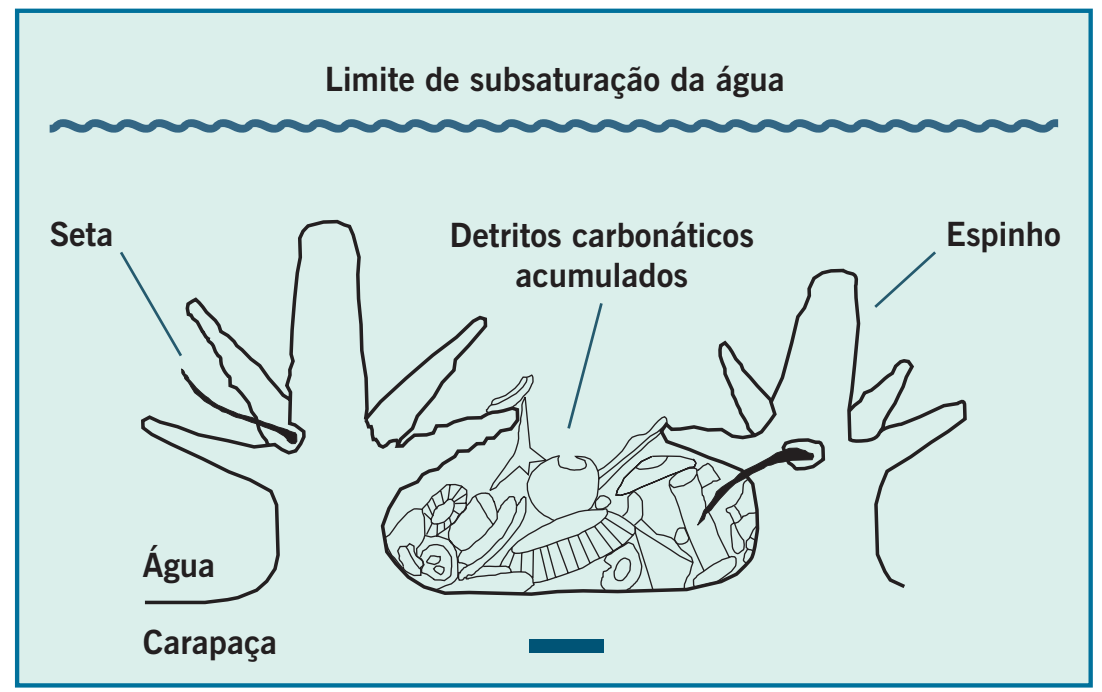

Figura 3 - Detalhe da superfície externa da carapaça mostrando o efeito dos detritos acumulados na ornamentação no afastamento da zona de subsaturação de carbonato (modificado de Swanson 1995). Escala: $5 \mu \mathrm{m}$
(1999) destacam a influência deste parâmetro na distribuição das espécies na plataforma equatorial brasileira, registrando assembléias diferentes conforme a composição sedimentar. As faunas de ostracodes neríticos diferem substancialmente das batiais e abissais (referidas como de águas profundas), pois além de possuírem populações menores, alguns taxa tendem a apresentar distribuição pandêmica. Em um transecto entre a plataforma continental e o sopé são encontradas diferentes associações, onde algumas espécies são restritas a determinadas batimetrias e outras têm distribuição mais ampla (euribáticas). Os eventos transgressivos e regressivos causam mudanças na posição da termoclina e desencadeiam migrações verticais de espécies ao longo do talude. Por esse motivo mudanças na composição faunística podem fornecer subsídios para a identificação de variações relativas do nível do mar.

As pesquisas sobre faunas de ostracodes de águas rasas e profundas se desenvolveram em ritmos diferentes, pelo fato da última demandar técnicas de coleta mais elaboradas e onerosas. Por isso, até o estabelecimento do DSDP, do ODP e projetos oceanográficos subseqüentes, os pioneiros nesta linha de pesquisa estudaram amostras de sondagem do fundo oceânico obtidas para fins diversos. Um bom exemplo é o estudo de Tressler (1941) realizado com um transecto de amostras coletadas entre a América do Norte e a Europa visando a viabilidade de instalação de cabos submarinos. O trabalho envolveu aspectos geológicos e biológicos dos sedimentos, inclusive sobre os ostracodes de águas profundas. Dentre as constatações mais relevantes daquele estudo pioneiro encontram-se o registro de taxa árticos e mediterrâneos e a baixa abundância de espécies. Alguns anos mais tarde Benson (1969) elaborou uma síntese do conhecimento existente até então sobre o tema que serviu de base para uma série de estudos 
desenvolvidos posteriormente por ele e outros autores. A partir de então o número de publicações a respeito de ostracodes de águas profundas cresceu consideravelmente, abrindo uma nova janela para a compreensão dos oceanos.

Estudos realizados nas últimas décadas trouxeram uma nova percepção sobre a distribuição geográfica destas faunas, onde foram avaliadas variáveis de natureza histórica e ecológica (Rex et al. 1993). Desta forma os fatores históricos envolvem a proximidade de centros de origem e os processos de dispersão entre os mesmos. Existe uma forte semelhança entre as faunas do Atlântico, Índico e Pacífico, que apontam este último como centro de origem de muitos taxa. Neste processo, even-

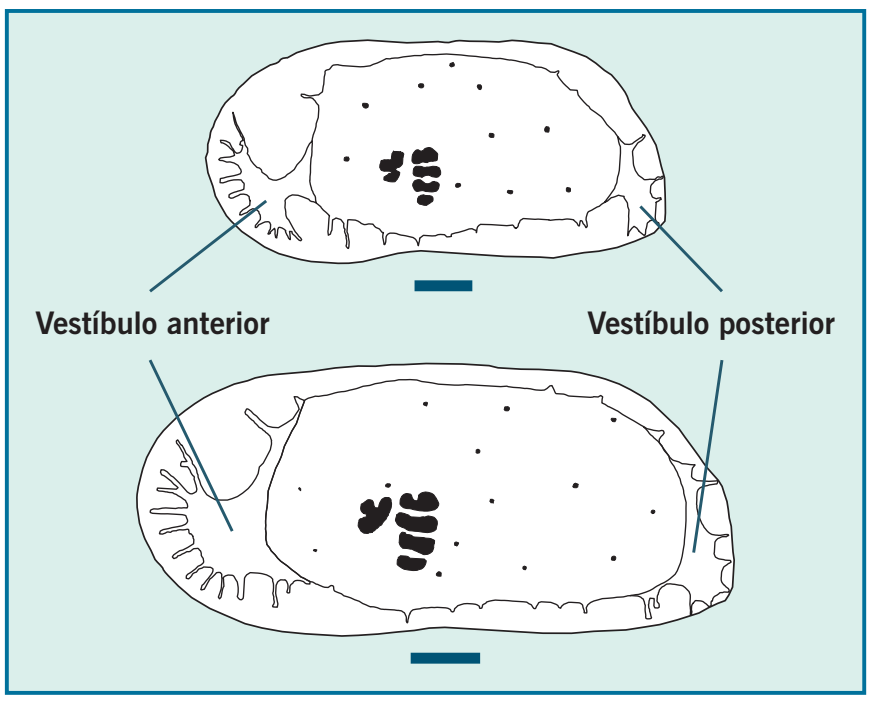

Figura 4 - Morfotipos de Krithe producta em vista interna mostrando a variação na forma do vestíbulo anterior. Escala: $100 \mu \mathrm{m}$ tos como a formação do istmo do Pa-

namá e a separação entre a América e a Antártica contribuíram para o estabelecimento de rotas de migração de espécies durante o Cenozóico (Whatley e Ayress 1988; Wood et al. 1999).

Os fatores ecológicos, por sua vez, relacionamse à ocorrência de determinadas espécies em resposta a condições favoráveis surgidas, como o aporte de recursos alimentares e mudanças na temperatura da água. Alguns trabalhos mostraram que em ambientes oligotróficos a diversidade é grande, embora a abundância seja pequena, enquanto nos eutróficos esta relação inverte-se (Van Harten, 1999). Os ostracodes são crustáceos que apresentam diferentes adaptações fisiológicas ao ambiente, o que explica a abundância de certos taxa em determinados contextos oceanográficos, conforme verificado em estudos como Cronin 1983, Dingle et al. 1990, Corrège 1993, Didié et al. 2002, Ayress et al. 2004 e Mazzini 2005, para mencionar apenas alguns exemplos. Trabalhos nesta linha permitiram a identificação de taxa restritos a grandes profundidades ou águas frias (criofílicos), importantes para caracterização paleoambiental. O gênero Krithe, por exemplo, é notadamente abundante e bem distribuído em bacias oceânicas profundas (Coles et al. 1994).

A rigor não existem feições morfológicas restritas a ostracodes batiais ou abissais, sendo o tubérculo ocular a característica mais importante na diagnose de espécies alóctones em uma asssociação, pois os ostracodes de águas profundas são cegos e desprovidos desta estrutura. Esforços foram realizados na busca de eventuais aspectos morfológicos que apresentassem uma aplicação paleoambiental. Peypouquet (1979) associou o tamanho e a forma do vestíbulo (estrutura existente entre a lamela externa e a duplicatura em alguns ostracodes) de espécies de Krithe com os níveis de oxigênio. Segundo aquela hipótese, espécies com vestíbulos anteriores grandes habitariam águas com baixas concentrações de oxigênio dissolvido, enquanto vestíbulos pequenos ou pouco desenvolvidos habitariam águas mais oxigenadas (Fig. 4).

Esta idéia, segundo alguns autores (Whatley e Quanhong 1993) é inconsistente quando analisada sob os dados disponíveis para outras espécies de ostracodes e invertebrados marinhos. Este fato comprova que a variabilidade existente entre os vestíbulos de espécies simpátricas de Krithe, é conseqüência de fatores ainda não totalmente compreendidos.

\section{Os ostracodes e a evolução dos oceanos}

Estudos recentes têm apontado bons resultados mediante a utilização de ostracodes na detecção de eventos oceanográficos. O amplo registro fóssil e a sensibilidade a parâmetros ambientais torna o grupo um potencial indicador de mudanças oceânicas, especialmente no Cenozóico. Os oceanos são sistemas dinâmicos que sofreram variações de nível locais e globais, expressas por meio de transgressões e regressões marinhas. Variações globais, 
denominadas glácio-eustáticas, são mais rápidas e significativas e refletem a formação e derretimento de massas de gelo em altas latitudes. Já as locais podem resultar de processos tectônicos ou sedimentares nas bacias marginais.

Estes eventos apresentam registros geológicos na forma de estruturas sedimentares, e/ou conteúdo fossilífero. O Quaternário constitui um período da história da Terra extremamente rico em registros desta natureza, onde a aplicabilidade dos ostracodes é enorme. Nestes ciclos transgressivos-regressivos, a posição da termoclina e da zona de mínimo de oxigênio a ela associada muda ao longo do talude (Fig. 5), provocando a migração de ostracodes entre diferentes batimetrias e mudanças de diversidade na alternância de períodos glaciais e interglaciais (Cronin e Raymo 1997; Rodriguez-Lázaro e Cronin 1999).

Os ostracodes auxiliam também a compreender o processo de estruturação dos oceanos, corroborando estudos realizados com base em outros grupos fósseis. Analisando densidades populacionais e sucessões de faunas a partir de uma base de dados global Benson (1990) identificou cinco grandes eventos últimos 80 Ma registrados nas assembléias de ostracodes. De acordo com aquele autor, o evento de extinção em massa ocorrido entre o Cretáceo e Paleógeno teve um efeito negativo nas faunas de ostracodes marinhos, onde estima-se que 15\% dos gêneros tenham se extingüido. No Eoceno, a abertura de portais oceânicos como o existente entre a Antártida e a Austrália, afetou o sistema de circulação oceânica, desencadeando o estabelecimento global da psicrosfera. A partir de então, as regiões polares passaram a ser pontos de afundamento de águas oriundas da região equatorial e distribuição de águas frias para as demais bacias oceânicas.

Ao longo do Mioceno ocorreu o fechamento da parte leste do mar de Tethys, gerando um au- mento de abundância e diversidade das faunas. No final dessa época, um máximo glacial e a "Crise de Salinidade do Messiniano" decorrente do isolamento do Mediterrâneo, voltaram a afetar os ostracodes. Finalmente, o soerguimento do istmo do Panamá há 3,5 Ma, promoveu o fechamento de um importante portal entre o Pacífico e o Atlântico, afetando a composição faunística dos dois oceanos. Além das migrações, estudos realizados sugerem que mudanças ambientais podem também modular a evolução, rompendo períodos de estase e desencadeando o surgimento de novos taxa (Benson, 1983).

Antes da estratificação térmica dos oceanos as picnóclinas eram determinadas basicamente por gradientes de salinidade e menos restritivas à migração dos ostracodes entre diferentes batimetrias. Quando os gradientes térmicos estabeleceram-se, faunas derivadas de taxa de águas rasas evoluíram com certo isolamento, formando as que hoje habitam a psicrosfera. Os eventos acima citados não são registrados em todas as bacias oceânicas, sugerindo que tiveram diferentes intensidades conforme a região considerada (Majoran e Dingle 2002).

Além de mudanças do nível relativo do mar, os eventos glaciais afetam também a circulação, produtividade primária e sedimentação oceânica. Variações eustáticas estão em geral associadas a maior incidência de processos turbidíticos, os quais têm maior probabilidade de ocorrer durante regressões ou transgressões marinhas (Weaver e Kuipjers 1983). A causa das glaciações atrai há muito tempo a atenção dos pesquisadores que desde cedo perceberam sua ciclicidade e a forte influência que exercem no clima terrestre. Contudo, foi só na primeira metade do século XX que o cientista sérvio Milatun Milankovitch propôs uma teoria relacionando-as com oscilações orbitais. Estas oscilações ocorrem ciclicamente, afetando a precessão dos
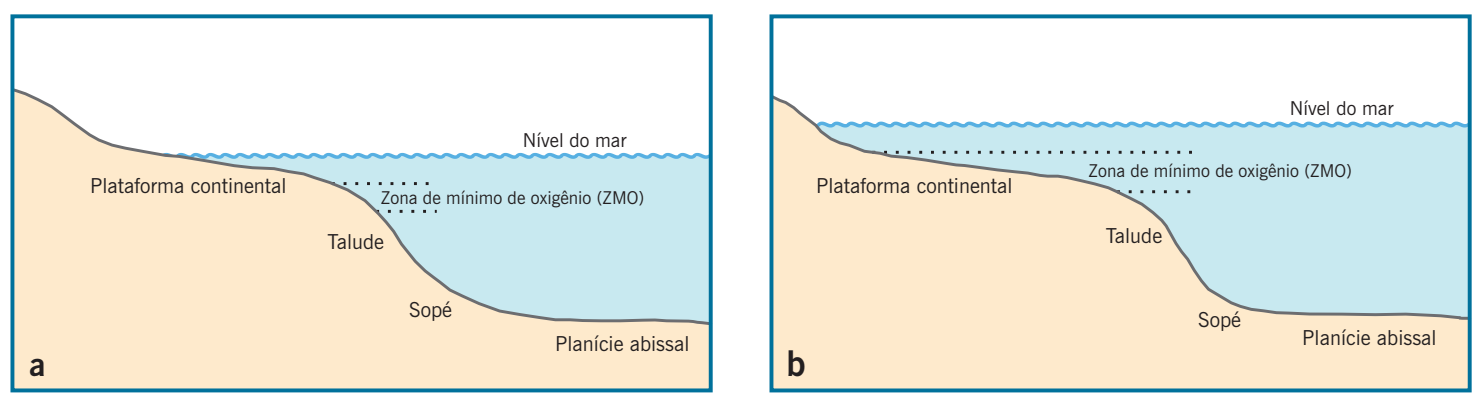

Figura 5 - Migração da zona de mínimo de oxigênio durante os eventos de variação do nível do mar. A. Evento regressivo; B. Evento transgressivo. 
equinócios, a obliqüidade e a excentricidade da órbita terrestre causando variações na intensidade da insolação em altas latitudes.

Os processos turbidíticos são importantes no estudo de faunas de águas profundas, pois formam associações mistas, compostas pela mistura de espécies autóctones e alóctones. Whatley (1988), propôs um modelo para identificação de elementos alóctones por meio da análise da estrutura populacional, permitindo distinguir biocenoses e tanatocenoses por meio da razão entre adultos e juvenis. Este dado pode ser complementado com o estado de preservação das carapaças (indicativo de transporte post mortem) e o conhecimento prévio da distribuição batimétrica das espécies. Deste modo elementos alóctones podem ser identificados com maior segurança, permitindo um zoneamento batimétrico mais acurado e aumentando a potencialidade de utilização das associações em estudos paleoambientais.

Em um outro tipo de abordagem, em vez de associações como um todo, determinados gêneros são utilizados isoladamente no estudo da evolução dos oceanos. Além do gênero Krithe, discutido previamente, há um grupo de ostracodes denominados citerelídeos que são também apontados por alguns pesquisadores como indicadores dos níveis de oxigênio do meio. Diversos autores têm observado o aumento da abundância destes ostracodes na zona de mínimo de oxigênio, e em intervalos correspondentes a eventos disaeróbicos ocorridos no passado.

Acredita-se que a relação entre os níveis de oxigênio e os picos de abundância dos citerelídeos está relacionada com suas características morfológicas. Diferente da maioria dos ostracodes bentônicos que são detritívoros ou predadores, estes têm hábito alimentar filtrador. O batimento das placas branquiais dos apêndices intensifica a circulação de água no interior da carapaça, otimizando tanto a filtração como as trocas gasosas. Whatley (1995) apontou ainda a estratégia reprodutiva do grupo como uma vantagem adicional, pois incubando seus instars no interior da carapaça, minimizam o efeito das baixas concentrações de oxigênio. Esta hipótese foi reforçada com o estudo de Corbari et al. (2005), que constatou estas adaptações por meio da análise morfo-funcional de uma espécie do gênero. Contudo, este método é aceito com ressalvas por alguns pesquisadores, que sustentam que picos de abundância de citerelídeos podem ser resultantes de outros fatores, conforme discutido por Swanson (2005).
Somados aos estudos faunísticos, análises geoquímicas vêm sendo progressivamente empregadas, tanto de elementos-traço como isótopos estáveis, cujas proporções nas carapaças são influenciadas por variáveis ambientais. $\mathrm{O}$ emprego destas metodologias mudou paradigmas da micropaleontologia marinha, como o de que os ecossistemas oceânicos profundos seriam estáveis devido ao seu isolamentodos das mudanças climáticas. Contudo, a integração de dados faunísticos e geoquímicos permitiu constatar que mesmo eventos de pequena duração afetam estas regiões (Cronin et al. 1999).

As análises geoquímicas começaram a ser aplicadas à micropaleontologia na primeira metade do século XX e difundiram-se a partir da década de 1950, a medida em que as técnicas foram aprimoradas. Abaixo são apresentadas generalidades sobre sua aplicação em ostracodes marinhos com base em resultados de diversos autores.

\subsection{Os elementos-traço}

As carapaças dos ostracodes são formadas por meio de reações envolvendo substâncias captadas do ambiente e do próprio organismo. Chamamos elementos-traço os elementos presentes na água incorporados nas carapaças durante sua precipitação. Desta forma, o aumento da razão molar de um elemento representa a maior disponibilidade do mesmo ou a existência de uma condição que incremente sua precipitação. Os principais elementos-traço analisados em ostracodes são o magnésio $(\mathrm{Mg})$ e o estrôncio $(\mathrm{Sr})$, cuja quantificação permite estimar a temperatura e a salinidade do ambiente.

Dentro desta categoria, a análise da razão $\mathrm{Mg}$ / Ca como paleotermômetro é a mais difundida, sendo os gêneros Krithe e Loxoconcha os mais utilizados em estudos paleoceanográficos de águas profundas e rasas, respectivamente. O método fundamenta-se no fato da quantidade de $\mathrm{Mg}$ incorporado ser influenciada positivamente pela temperatura da água, conforme a relação $(\mathrm{Mg} / \mathrm{Ca})_{\text {calcita }}=$ $\mathrm{D}(\mathrm{Mg} / \mathrm{Ca})_{\text {agua }}$, sendo $\mathrm{D}$ o coeficiente de distribuição, o qual é definido pela equação:

$$
\mathrm{D}_{(\mathrm{Mg})}=[(\mathrm{Mg} / \mathrm{Ca}) \text { ostracode } /(\mathrm{Mg} / \mathrm{Ca}) \text { água }]
$$

A quantificação do Mg na carapaça é obtida por meio de espectrometria de emissão, e o valor obtido é convertido em paleotemperaturas por meio de um cálculo que fornece a temperatura da água 
na qual a carapaça formou-se. Inicialmente, os equipamentos limitavam a análise a um único elemento, mas o advento do ICPMS (Inductively Coupled Plasma Mass Spectrometry) permitiu a análise simultânea de diferentes elementos com o uso de uma única carapaça (Rosenthal et al. 1999). O uso de valvas inteiras e limpas é aconselhável, uma vez que os elementos não estão distribuídos homogeneamente na carapaça e contaminantes podem afetar os resultados (Cronin et al. 2005). Uma descrição mais detalhada sobre a análise de $\mathrm{Mg} / \mathrm{Ca}$ em ostracodes marinhos e os processos de preparação dos espécimes é encontrada em Dwyer et al. (2002).

Devido ao seu grande tempo de residência, assume-se que a concentração de $\mathrm{Mg}$ nos oceanos seja constante numa escala de tempo de milhões de anos, sendo a incorporação regulada apenas pelo coeficiente de distribuição. Embora se saiba que a quantidade de $\mathrm{Mg}$ co-precipitado varie conforme o gênero e o estágio ontogenético, os fatores que explicam isto não são ainda satisfatoriamente compreendidos, o que requer ajustes metodológicos para sua utilização em diferentes taxa (Dwyer et al. 2002; Kondo et al. 2005).

\subsection{Os isótopos estáveis}

Isótopos são átomos de um mesmo elemento que apresentam diferentes números de nêutrons em seus núcleos, portanto, diferentes massas atômicas. Recebem este nome porque a configuração do núcleo não varia ao longo do tempo por meio de decaimento radioativo.

O sinal isotópico de um carbonato pode fornecer informações sobre temperatura, volume de gelo e circulação de um oceano. Sua utilização fundamenta-se no trabalho de Urey (1947), que demonstrou que carbonatos precipitados em uma mesma solução apresentam diferentes razões entre ${ }^{18} \mathrm{O}$ e ${ }^{16} \mathrm{O}$, conforme a temperatura. Contudo, sua aplicação consagrou-se com o trabalho de Emiliani (1955), que utilizando esta técnica em foraminíferos planctônicos corroborou o modelo de ciclicidade das variações orbitais.

Com base nisto, o autor propôs a subdivisão do Quaternário em estágios isotópicos marinhos (EIM) representados por uma seqüência numérica, onde os números pares correspondem aos períodos glaciais, e os ímpares aos interglaciais. Neste modelo, o interglacial atual é representado pelo número 1, o último máximo glacial (UMG) pelo número 2, e assim sucessivamente. Aqueles resultados foram corroborados por estudos posteriores onde curvas isotópicas montadas exibiram padrões similares permitindo, inclusive, a subdivisão dos EIM (Pisias et al. 1984).

A razão isotópica em uma amostra é representada pela letra grega $\delta$ e expressa em partes por mil (\%o). A abundância natural do isótopo ${ }^{16} \mathrm{O}$ é de $99,76 \%$ e a do isótopo ${ }^{18} \mathrm{O}$ é de $0,19 \%$, porém, estas proporções oscilam entre períodos glaciais e interglaciais. Durante os glaciais a água do mar encontra-se enriquecida com o isótopo mais pesado, o ${ }^{18} \mathrm{O}\left(\right.$ picos positivos $=$ enriquecimento de $\left.{ }^{18} \mathrm{O}\right)$. Já a água depositada nas geleiras, encontra-se depletada de ${ }^{18} \mathrm{O}$ e enriquecida com o isótopo mais leve, $\mathrm{O}{ }^{16} \mathrm{O}$. Isso ocorre porque o isótopo leve é extraído mais facilmente por meio da evaporação. A água evaporada migra em direção a altas latitudes e precipita-se, ficando retida nas geleiras. Ao término dos períodos glaciais, o derretimento em larga escala destas massas de gelo promove o enriquecimento da água com ${ }^{16} \mathrm{O}$ (picos negativos de ${ }^{18} \mathrm{O}$ ) restabelecendo a proporção natural entre os dois isótopos.

A razão molar de um isótopo numa carapaça pode refletir ou não a razão isotópica da água: quando a razão do carbonato e a da água são as mesmas, diz-se que a precipitação ocorreu em equilíbrio, e quando diferem diz-se que ocorreu em desequilíbrio. Esta diferença, chamada efeito vital, decorre de peculiaridades metabólicas dos diferentes grupos.

A razão isotópica em carbonatos é obtida por meio de espectrômetros de massa, sendo as carapaças dissolvidas em uma solução de ácido fosfórico e $\mathrm{CO}_{2}$ liberado nesta reação analisado. A razão obtida é comparada com um padrão pré-estabelecido, geralmente o PDB ou SMOW, conforme a seguinte equação:

$$
\begin{aligned}
& \delta{ }^{18} \mathrm{O}=\left(\mathrm{R}_{\text {amostra }}-\mathrm{R}_{\text {padrāo }}\right) \cdot 10^{3} / \mathrm{R}_{\text {padrão }} \\
& \text { onde, } \mathrm{R}={ }^{18} \mathrm{O} /{ }^{16} \mathrm{O}
\end{aligned}
$$

As primeiras aplicações de análises isotópicas em ostracodes marinhos, como a de Durazzi (1977) não apresentaram resultados conclusivos. Estudos mais recentes (Didiè e Bauch 2002, Mazzini 2005) têm difundido sua aplicação, mostrando que a razão isotópica apresenta um desvio em relação a água que não a invalida, contudo, como um recurso adicional em estudos paleoambientais. 
Alguns autores sugerem que a combinação de análises isotópicas e de elementos traço fornece resultados mais consistentes a um estudo (Lear $e t$ al. 2000). Isto explica-se pelo fato da razão $\mathrm{Mg} / \mathrm{Ca}$ não ser alterada pela formação das massas de gelo, refletindo essencialmente a influência da temperatura no momento da precipitação do carbonato.

\section{A pesquisa paleoceanográfica com base em ostracodes no Brasil}

As potencialidades apresentadas pelos ostracodes, algumas das quais foram discutidas neste texto, torna-os um dos grupos de microfósseis mais estudados na atualidade. No Brasil este campo de pesquisa tem se desenvolvido nos últimos anos, contribuindo para o conhecimento oceanográfico tanto sob o aspecto biológico quanto geológico.

O início da ostracodologia brasileira remonta ao final do século XIX quando o naturalista britânico George S. Brady publicou em 1880 um estudo sobre ostracodes marinhos coletados na expedição do Challenger, a qual abrangeu parte da costa nordeste do país. Porém, foi aproximadamente um século mais tarde, com o levantamento preliminar da fauna em nível genérico realizado por Pinto et al. (1978), que o estudo do grupo no Brasil foi efetivamente iniciado.

Os primeiros estudos com escopo paleoceanográfico foram publicados na década de 1970, abrangendo especialmente a identificação e distribuição das espécies de águas profundas. $\mathrm{O}$ artigo publicado por Benson (1977), envolvendo a região sudeste da margem continental foi o precursor do estudo sistemático dos ostracodes batiais brasileiros. Naquele trabalho, onde estudou amostras da pernada 39 do DSDP, abrangendo o Maastrichtiano e o Terciário, foram propostos dois gêneros e dez novas espécies. Este estudo identificou mudanças faunísticas significativas que ampliaram o conhecimento sobre os ostracodes batiais no Atlântico Sul e a resposta das faunas aos eventos ocorridos. 
Posteriormente, Benson e Peypouquet (1983) estudaram amostras da pernada 72 do DSDP, na Elevação de Rio Grande, que abrangiam o intervalo entre o Maastrichtiano e o Quaternário. Além da descrição de novas espécies, este trabalho permitiu igualmente a observação de mudanças faunísticas em consonância com variações hidrológicas.

Os ostracodes de águas profundas ficaram algum tempo sem ser pesquisados até que Carmo e Sanguinetti $(1995,1999)$ estudaram a taxonomia e ecologia do gênero Krithe, identificando a presença de nove espécies e avaliando a influência das massas d'água na distribuição batimétrica das mesmas. Com base nas associações faunísticas da porção sul da margem continental, aqueles autores propuseram o estabelecimento da Corrente das Malvinas durante o Mioceno.

Um estudo dessa natureza foi também realizado na bacia de Pelotas, sul do Brasil, por Carreño et al. (1999). Aquele trabalho abordou a fauna de ostracodes neoterciários e quaternários, sob o aspecto sistemático e permitiu relacionar as mudanças faunísticas com os eventos transgressivos e regressivos registrados naquela bacia.

Mais recentemente foi iniciado o estudo das faunas quaternárias batiais da bacia de Santos, região sudeste do Brasil (Fig. 6). Por meio de análises faunísticas e geoquímicas, foram observadas mudanças nas assembléias durante nos últimos 30000 anos atribuídas a eventos climáticos ocorridos neste período (Bergue et al. 2006). Destes, o Último Máximo Glacial (UMG) e a transição para o interglacial holocênico foram os mais significativos, conforme demonstrado pelo surgimento e desaparecimento local de espécies e mudanças de abundância. Um outro resultado interessante deste trabalho é a constatação da ocorrência de espécies até então registradas apenas no Pacífico sul, revelando que eventos possivelmente estabeleceram rotas de migração entre estas duas regiões.

Conforme apresentado nesta breve revisão, o estudo das assembléias de ostracodes constitui um excelente auxiliar na pesquisa paleoceanográfica, especialmente em termos de mudanças hidrológicas. Pesquisas futuras nesta área poderão agregar novos conhecimentos sobre o estabelecimento de correntes oceânicas e mudanças nas massas d'água, auxiliando a compreender a influência das mudanças climáticas nos ecossistemas marinhos.

\section{Agradecimentos}

O autor manifesta seus agradecimentos a Gerson Fauth e demais revisores anônimos pela leitura crítica e sugestões apresentadas para a melhoria deste texto.

\section{Referências bibliográficas}

Armstrong H.A., Brasier M.D. 2005. Microfossils. Blackwell Publishing. 296p.

Ayress M.A., De Deckker P., Coles G. 2004. A taxonomic and distributional survey of marine benthonic Ostracoda off Kerguelen and Heard islands, South Indian Ocean. J. Micropalaeont., 23:15-38.

Benson R., Peypouquet J. P. 1983. The upper and mid-bathyal cenozoic ostracode faunas of the Rio Grande rise found on Leg 72 Deep Sea Drilling Project. Initial Reports DSDP, 62:805-818.

Benson R. 1972. The Bradleya problem, with description of two new psychrospheric ostracode genera, Agrenocythere and Poseidonamicus (Ostracoda: Crustacea). Smithson. Contrib. Paleobiol., 12:1-138.

Benson R. 1977. The Cenozoic ostracode faunas of the São Paulo Plateau and Rio Grande Rise (DSDP LEG 39, sites 356 and 357). Initial Reports DSDP, 39:856-883.

Benson R. 1983. Biomechanical stability and sudden change in the evolution of the deep-sea ostracode Poseidonamicus. Paleobiology, 9(4):398-413.

Benson R. 1990. Ostracoda and the discovery of global Cainozoic palaeoceanographical events. In: Whatley R., Maybury C. ed. Ostracoda and Global Events. Londres: Chapmann and Hall, 1990. p. 41-58.

Benson R. 1969. Preliminary reports on the study of abyssal ostracods. In: Neale, J. ed. The Taxonomy, Morphology and Ecology of Recent Ostracoda. Edinburgh: Oliver \& Boyd. p. 475-480.

Bergue C.T, Costa K.B., Dwyer G., Moura C. 2006. Bathyal ostracode diversity in the Santos Basin, Brazilian southeast margin: response to Late Quaternary climate changes. Rev. Bras. Paleont., 9(2):201-210.

Bradley R.S. 1999. Paleoclimatology - reconstructing climates of the Quaternary. International Geophysics Series, volume 68. 614p.

Brady G. 1880. Report on the Ostracoda dredged by H. M. S. Challenger during the years 1873-76. Report of Scientific Results of the Voyage of H. M. S. Challenger - Zoology, 1:1-184. 
Bruun, A.F.1955. Deep sea and abyssal depths. Memoir geological Society of America, 67(1):641-672.

Carmo D.A., Sanguinetti Y. 1999. Taxonomy and palaeoceanographical significance of the genus Krithe (Ostracoda) in the Brazilian margin. $J$. Micropalaeont., 18:111-123.

Carmo D.A., Sanguinetti Y. 1995. Krithe occurrence on the Brazilian continental margin - an ecological approach. In: Riha J. ed. Ostracoda and Biostratigraphy. Rotterdam: Balkema. p. 407-412.

Carreño A.L., Coimbra J.C., Carmo D.A. 1999. Late Cenozoic sea level changes evidenced by ostracodes in the Pelotas Basin, southernmost Brazil. Mar. Micropaleontol., 37:117-129.

Coimbra J.C., Pinto I.D., Würdig N.L., Carmo D.A. 1999. Zoogeography of Holocene Podocopina (Ostracoda) from the Brazilian equatorial margin. Mar. Micropaleontol., 37:365-379.

Coles G., Whatley R., Moguilevsky A. 1994. The ostracode genus Krithe from the Tertiary and Quaternary of the North Atlantic. Palaeontology, 37(1):71-120.

Corbari L., Mesmer-Dudons N., Carbonel P., Massabuau J.-C. 2005. Cytherella as tool to reconstruct deep-sea paleo-oxygen levels: the respiratory physiology of the platycopid ostracod Cytherella cf. abyssorum. Mar. Biol., 147(6):13771386.

Corrège T. 1993. The relationship between water masses and benthic ostracod assemblages in the western Coral Sea, Southwest Pacific. Palaeogeogr. Palaeoclimatol. Palaeoecol., 105:245-266.

Cronin T., Raymo M.E.1997. Orbital forcing of deep-sea benthic species diversity. Nature, 385:624-626.

Cronin T. 1983. Bathyal ostracodes from the FloridaHatteras slope, the straits of Florida, and the Blake Plateau. Mar. Micropaleontol., 8:89-119.

Cronin T.M., De Martino D.M., Dwyer G.S., Rodriguez-Lázaro J. 1999. Deep sea ostracode species diversity: response to late Quaternary climate change. Mar. Micropaleontol., 37:231-249.

Cronin T.M., Kamiya T., Dwyer G.S., Belkin H., Vann C.D., Schwede S., Wagner R. 2005. Ecology and shell chemistry of Loxoconcha matagordensis. Palaeogeogr. Palaeoclimatol. Palaeoecol.,. 225:14-67.

Didié C., Bauch H.A. 2002. Implications of Upper Quaternary stable isotope records of marine ostracodes and benthic foraminifers for palaeocological and palaeoceanographical investigations. In: J.A. Holmes, A.R. Chivas. ed. The Ostracoda: applications in Quaternary research. American Geophysical Union, p. 279301. (Geophysical Monograph 131).

Didié C., Hening A.B., Helmke J.P. 2002. Late Quaternary deep-sea ostracodes in the polar and subpolar North Atlantic: paleoecological and paleoenvironmental implications. Palaeogeogr. Palaeoclimatol. Palaeoecol., 184:195-212.

Dingle R., Lord A.R., Boomer I. 1990. Deep-water quaternary Ostracoda from the continental margin off south-western Africa (SE Atlantic Ocean). Ann. S. Afr. Mus., 99(9):245-366.

Durazzi J. 1977. Stable isotopes in the ostracod shell: a preliminary study. Geoch. Cosmoch. Acta, 41:1168-1170.

Dwyer G., Cronin T. M., Baker P.A., Raymo M.E., Buzas J.S., Corrège T. 1995. North Atlantic deepwater temperature change during Late Pliocene and Late Quaternary climatic changes. Science, 270:1347-1351.

Dwyer G.S., Cronin T.M., Baker P.A. 2002. Trace elements in marine ostracodes. In: J.A. Holmes, A. Chivas. eds. The Ostracoda. Applications in Quaternary Research, American Geophysical Union, p. 205-226. (Geophysical Monograph 131).

Emiliani C. 1955. Pleistocene temperatures. J. Geol, 63:538-578.

Hunt G., Roy K. 2006. Climate change, body size evolution, and Cope's Rule in deep sea ostracodes. PNAS, 103(5):1347-1352.

Lear C.H., Elderfield H., Wilson P.A. 2000. Cenozoic deep-sea temperatures and global ice volumes from $\mathrm{Mg} / \mathrm{Ca}$ in benthic foraminiferal calcite. Science, 287:269-272.

Majoran S., Dingle R. 2002. Cenozoic deep-sea ostracods from Maud Rise, Weddel Sea, Antarctica (ODP site 689): a palaeoceanographical perspective. Geobios, 35:137-152.

Mazzini I. 2005. Taxonomy, biogeography and ecology of Quaternary benthic Ostracoda (Crustacea) from circumpolar deep water of the Emerald basin (Southern Ocean) and the $S$ Tasman Rise (Tasman Sea). Senckenbergiana Maritima, 35(1):1-119.

Peypouquet J.-P. 1979. Ostracodes et paléoenvironements. Methodology et application aux domains profonds du Cenozóique. Bulletin Burean Recherche Geologie et Mineralogie, 4(1):3-79. 
Pinto I.D., Ornellas L.P., Purper I, Kotzian S.B., Sanguinetti Y.T. 1978. Recent ostracodes along $7408 \mathrm{Km}$ of Brazilian coast ( $33^{\circ} 45^{\prime} \mathrm{S}$ to $\left.04^{\circ} 25^{\prime} \mathrm{N}\right)$. Pesquisas, 9:109-120.

Pisias N.G, Martinson D.G., Moore Jr. T.C., Shackleton N.J., Prell W., Hays J., Boden G. 1984. High resolution stratigraphic correlation of benthic oxygen isotopic records spanning the last 300000 years. Mar. Geol., 56:119-136.

Rex M.A., Suart C.T., Hessler R.R., Allen J.A., Sanders H.L., Wilson G.D.F. 1993. Global scale latitudinal patterns of species diversity in the deep-sea benthos. Nature, 365:636-639.

Rodriguez-Lazaro J., Cronin T. 1999. Quaternary glacial and deglacial Ostracoda in the thermocline of the Little Bahama Bank (NW Atlantic): palaeoceanographic implications. Palaeogeogr. Palaeoclimatol. Palaeoecol., 152:339-364.

Rosenthal Y., Field M.P., Sherrell R.M. 1999. Precise determination of element/calcium ratios in calcareous simples using sector field inductively coupled plasma mass spectrometry. Anal. Chem., 71(15):3248-3253.

Seibold E., Berger W. 1996. The sea floor - an introduction to marine geology. Springer. 339p.

Swanson K. M. 1995. Strategies for and evolutionary implications of carapace conservation in some living benthic marine Ostracoda. Senckenbergiana Lethaea, 75(1/2):193-213.

Swanson K.M., Jellinek T., Malz H. 2005. The platycopine condition: new observations on reproduction, respiration and feeding in living, deep-sea Platycopina (Crustacea: Ostracoda). Senckenbergiana Maritima, 35(2):157-187.
Tressler W. 1941. Geology and biology of North Atlantic deep-sea cores between Newfoundland and Ireland, Part IV, Ostracoda. Denver: U. S. Geological Survey, p. 95-106. (USGS Prof. Paper, 169C).

Urey H.C. 1947. The thermodynamic properties of isotopic substances. Journal of Geochemical Society, p. 562-581.

Van Harten D. 1999. Origin and diversity of cainozoic deep-sea Ostracoda. Crustaceana, 72(8):993-998.

Weaver P.P.E., Kuipjers A. 1983. Climatic control of turbidite deposition on the Madeira Abyssal Plain. Nature, 306:360-363.

Whatley R., Ayress M. 1988. Pandemic and endemic distribution patterns in Quaternary deep-sea Ostracoda. In: Hanai T., Ikeya N., Ishizaki K. Evolutionary Biology of Ostracoda. Amsterdam: Elsevier. p. 739-755.

Whatley R., Quanhong Z. 1993. The Krithe problem: a case history of the distribution of Krithe and Parakrithe (Crustacea, Ostracoda) in the South China sea. Palaeogeogr. Palaeoclimatol. Palaeoecol., 103:281-297.

Whatley R. 1995. Ostracoda and oceanic palaeoxygen levels. Mittelungen Hamburg Zoologische Museum Institut, 92:337-353.

Whatley R. 1988. Population structure of ostracods: some general principles for the recognition of palaeoenvironments. In: De Deckker P., Colin J.-P., Peypouquet J.-P. ed. 1988. Ostracoda in the Earth Sciences. Amsterdam: Elsevier. p. 245-256.

Wood A.R., Ramos M.I.F., Whatley R.C. 1999. The paleozoogeography of Oligocene to Recent marine Ostracoda from the neotropics (mid- and South America). Mar. Micropaleontol., 37:347-364.

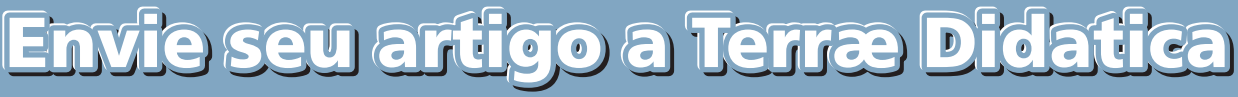

Terre Didatica convida pesquisadores e professores a enviarem contribuições de estudos, pesquisas e experiências de ensino de Geociências e Eđucação Ambiental em qualquer nível de ensino. Acesse http:||wwwige.unicamp.br/terraedidatical e veja os formatos e normas de publicação. 